\title{
A SITUAÇĀO DA CITOTAXONOMIA DE ANGIOSPERMAS NOS TRÓPICOS E, EM PARTICULAR, NO BRASIL
}

\author{
Marcelo Guerra'
}

\begin{abstract}
RESUMO - A citotaxonomia vegetal desenvolveu-se inicialmente nos países do primeiro mundo, acumulando dados e estabelecendo modelos evolutivos baseados principalmente em táxons e floras temperadas. A introdução de técnicas citogenéticas mais refinadas tem permitido uma análise mais acurada da variabilidade cariotípica, mas até o momento estes novos métodos têm sido aplicados apenas a um número muito pequeno de táxons. Assim, o principal instrumento do citotaxonomista continua sendo a variabilidade cromossômica numérica. A região tropical ainda é muito carente de dados, especialmente os trópicos americanos. No Brasil, a citotaxonomia se desenvolveu como parte da citogenética e não como uma abordagem taxonômica. Mesmo assim, nos últimos anos tem crescido o número de laboratórios trabalhando com citogenética vegetal e tem havido muitos avanços no estudo citotaxonômico de elementos de nossa flora.
\end{abstract}

Palavras-chave: Citotaxonomia, Citogenética, Citotaxonomia no Brasil.

\begin{abstract}
Plant cytotaxonomy was developed in Europe and W. America, accumulating data and establishing evolutionary models based mainly on temperate taxa and floras. The introduction of more refined cytogenetic techniques has allowed an improved analysis of the karyotypic variability, but these new methods have been applied only to a very small number of taxa. Consequently, the main focus of the cytotaxonomy is still the chromosome number. Chromosomal data for tropical species are still very scarce, especially those from the American tropics. In Brazil, cytotaxonomy has been developed as an adjunct of cytogenetics rather than as a taxonomic approach. However, in the recent years these has been an increase in the number of laboratories working on plant cytogenetics and many advances in cytotaxonomic studies on representatives of our flora have been made.
\end{abstract}

Key-words: Cytotaxonomy, Cytogenetics, Cytotaxonomy in Brazil.

\section{Introdução}

O uso de dados citogenéticos na taxonomia vêm sendo aplicado desde o início do século. A primeira observação nesse sentido provavelmente foi a de

1 - Universidade Federal de Pernambuco, CCB., Departamento de Biologia Geral, 50.739, Recife, PE, Brasil. 
Ferguson, 1904 (citado por Ehrendorfer, 1964), que mostrou que o gênero $\mathrm{Pi}$ nus podia ser caracterizado pela constância do número cromossômico básico $\mathrm{n}=12$. Nas duas a três décadas que se seguiram foi examinado um grande número de gêneros, com extensas análises populacionais a nível infra-específico. Esses trabalhos, que estabeleceram a base da citotaxonomia vegetal clássica, estão muito bem revisados por Böcher (1961) e Ehrendorfer (1964). A maioria desses trabalhos, no entanto, são relativos a grupos taxonômicos de regiões temperadas, principalmente da flora européia e norte-americana. Por esse motivo, Ehrendorfer (1970) chama a atenção para a possibilidade de que os mecanismos evolutivos ou as estratégias adaptativas a nível cariológico, reconhecidos nas plantas de regiōes temperadas, não sejam os mesmos ou não tenham o mesmo significado nas populações tropicais, especialmente nas espécies lenhosas. De fato, Stebbins (1966) enfatiza que o cariótipo de plantas tropicais difere em alguns aspectos importantes daquele de plantas temperadas (ver também Morton, 1966 e Levim \& Funderburg, 1979). Isso mostra a necessidade de se desenvolver um grande número de estudos citogenéticos em plantas tropicais, para tentar compreender o seu possível significado adaptativo e taxonômico. No presente trabalho é apresentado um quadro geral da situação da citotaxonomia de plantas tropicais e, em especial, da flora brasileira.

\section{Citotaxonomia clássica e moderna}

A quase totalidade dos trabalhos citotaxonômicos se enquadra dentro do que se chama de citotaxonomia clássica, que se caracteriza pela observação de cromossomos ou núcleos interfásicos corados com o uso de técnicas relativamente simples, como a técnica de Feulgen, que cora a cromatina de forma específica e estequiométrica, ou o Carmin ou Orceina acética. Com essa abordagem tem sido possível vários níveis de análise citotaxonômica. A mais simples delas consiste em determinar e comparar o número cromossômico em um grande número de espécies de um determinado táxon. Nesse caso, é freqüentemente possível reconhecer o número cromossômico ancestral do grupo e, a partir daí, as diferentes linhas evolutivas desenvolvidas dentro do mesmo, marcadas por um rastro de variações graduais envolvendo principalmente diploidias e poliploidias.

Quando o material é citologicamente favorável, com cromossomos grandes e pouco numerosos, é possível refinar a análise definindo o tamanho e a morfologia cromossômica ou o número e posição dos satélites. Essas mesmas técnicas permitem ainda analisar a meiose e verificar a estabilidade cromossômica do grupo, ou identificar híbridos e sua natureza. Exemplos desses tipos de análises estão em todos os livros básicos de citotaxonomia e biossistemática, como o de Darlington (1956), o de Stace (1984) ou o de Stebbins (1971), o texto geral mais importante em citogenética e citotaxonomia vegetal. O uso 
do núcleo interfásico como parâmetro citotaxonômico tem sido menos utilizado, mas em alguns casos tem se mostrado muito útil (veja por exemplo, Guer$\mathrm{ra}, 1987)$. O texto mais abrangente continua sendo o trabalho pioneiro de Delay (1949). O assunto foi recentemente revisado por Guerra (1985).

Com o desenvolvimento de métodos mais refinados de análise dos cromossomos ou da cromatina interfásica, vem sendo desenvolvida o que se poderia chamar de citotaxonomia moderna, que alia às técnicas clássicas um número variado de técnicas desenvolvidas ou melhoradas nas duas últimas décadas. Nesse caso, a caracterização do cariótipo é complementada pela determinação da quantidade de DNA característico de cada espécie, diferenciação longitudinal cromossômica (técnicas de bandeamento), hibridização "in situ" do DNA de diferentes espécies, análise comparativa de uma determinada fração do DNA, etc. O uso desses métodos está amplamente ilustrado em coletâneas de simpósios especializados como as do International Organization of Plant Biosystematics (Grant, 1984) ou as do Kew Chromosome Conference (Brandhan, 1988).

Embora muito mais específicos, esses novos métodos são também muito mais trabalhosos, dispendiosos, demandam tempo mais longo e a repetibilidade dos resultados, em alguns deles, é menor que a desejada. Por essa razão, apenas um pequeno número de gêneros e tribos tem sido analisado com essas abordagens (veja revisão em Greilhuber, 1984). Nesses casos, tem sido quase sempre constatado que a variabilidade cariotípica é muito maior que a até então observada, o que enfatiza a importância do uso, sempre que possível, desses novos procedimentos na citotaxonomia.

Apesar desses importantes progressos técnicos, o número cromossômico continua sendo o parâmetro mais utilizado na citotaxonomia vegetal e sobre o qual dispomos de maior número de dados em todas as floras (Raven, 1975). Além disso, a poliploidia, o fenômeno citogenético mais comum em plantas e talvez o mais importante para a sistemática (Jackson, 1976; Lewis, 1980), é prontamente reconhecida pela simples contagem cromossômica. O acesso a esses dados foi extremamente facilitado pela construção de listas de números cromossômicos que compilam a grande maioria dos relatos desde o começo do século até os anos recentes, publicados sucessivamente por Fedorov (1969), Moore $(1973,1974,1977)$ e Goldblatt $(1981,1984,1985,1988)$. Convém observar que a maioria desses dados são puros relatos citológicos sobre o número cromossômico de espécies de determinada flora, jardins botânicos ou mesmo de casos isolados. Apenas em um número relativamente pequeno de trabalhos é feita uma análise taxonômica com esses dados. Por essa razão, e pela alta demanda de trabalhos, alguns periódicos abriram um espaço próprio e permanente para publicação de listas de números cromossômicos (veja, por exemplo, a revista Taxon, o Informatore Botânico Italiano e o Boletim da Sociedade Broteriana, Série 2). Tais dados são muito importantes para caracterização das floras regionais e estudos citogeográficos, e de grande utilidade pa- 
ra o citotaxonomista que deseja fazer uma revisão de um grupo de ampla distribuição geográfica. Contudo, esse tipo de análise citogenética desvinculada de um projeto taxonômico, quer na forma-de listas de número cromossômico quer como artigos isolados, é o que apresenta mais freqüentemente erros de identificação botânica ou de interpretação citológica. Erros desse tipo, especialmente freqüentes em listas mais antigas, constituem um dos principais problemas na citotaxonomia (Merxmüller, 1970; Favarger, 1978).

\section{Situação da citotaxonomia nos trópicos em geral}

A flora dos trópicos, especialmente a das regiōes economicamente menos desenvolvidas, é ainda relativamente pouco estudada. Raven (1975), revisando os dados disponíveis para cada família das angiospermas, observou que das 354 famílias reconhecidas por Cronquist em 1968, apenas 44 não possuiam nenhum dado citogenético. Dessas, mais de um terço são pequenas famílias endêmicas na América Central e do Sul. Além disso, nas famílias de ampla distribuição geográfica é comum que os representantes tropicais, e especialmente neotropicais, formem grandes lacunas de dados, dificultando a interpretação da família como um todo.

O estudo citogenético das floras tropicais tem sido feito em parte por botânicos estrangeiros (americanos ou europeus) e em parte por pesquisadores locais. Uma fração considerável do que sabemos sobre a citogenética da flora africana, ou sul-americana, vem de coletas sistemáticas de material vivo ou fixado, feitas por botânicos estrangeiros. O material de espécies fixadas durante longas excursões resulta, em geral, em preparações citológicas de baixa qualidade. Ao contrário, as coleções vivas mantidas em casas-de-vegetação produzem excelentes resultados (veja, por exemplo, Grant, 1955).

Grupos locais de pesquisa em citotaxonomia surgiram predominantemente nas regiōes tropicais colonizadas principalmente por franceses ou ingleses, que possuem tradição em estudos citológicos. Assim, a flora australiana teve vários de seus táxons endêmicos, ou predominantemente australianos, analisados citotaxonomicamente por pesquisadores locais há mais de 30 anos atrás (veja, por exemplo, a citotaxonomia da tribo Boronieae por Smith-White, 1954; da família Casuarinaceae por Barlow, 1959 ou das Proteaceae por Johnson \& Briggs, 1963). Smith-White (1959) estabeleceu um modelo de evolução cromossômica para a flora australiana que se aplica a diversos grupos endêmicos e grupos não-endêmicos com representantes endêmicos. Na África e Ásia Tropical houve um menor desenvolvimento, mas mesmo assim foram feitos vários estudos extensos, como a análise comparativa de centenas de espécies da África Ocidental por Mangenot \& Mangenot (1962) ou do Norte do Saara por Reese (1957). Uma situação excepcional ocorre na Índia que tem o maior número de citogeneticistas e citotaxonomistas trabalhando nos trópi- 
cos (veja, por exemplo, os resumos do simpósio editado por Manna \& Sinha (1981). Algumas compilações extensas e importantes por suas análises comparativas, como a de Mehra (1976), que discute os resultados obtidos em espécies arbóreas e não-arbóreas, são quase indispensáveis para citotaxonomistas de outras regiōes tropicais.

Sem dúvida a situação mais precária é a da América Tropical. A maior parte dos estudos em citotaxonomia da flora neotropical foi feita por pesquisadores estrangeiros e está dispersa por um sem-número de pequenos trabalhos. Trabalhos extensos, como o de Diers (1961), que analisou 208 espécies da cordilheira andina do Peru, são muito raros. Em geral, a análise cromossômica tem sido feita por métodos clássicos e restrita à determinação do número cromossômico. Uma notável exceção é o extenso trabalho desenvolvido nas Comelinaceae, especialmente na tribo Tradescantieae (Jones \& Kenton, 1984). Contudo, essas espécies com cromossomos grandes, núcleos reticulados e distribuição geográfica no limite da zona tropical, se expandindo pelo Norte do México e Sul dos E.U.A., podem não ser um modelo adequado para a flora tropical. Nesse sentido, o trabalho de Morawetz (1986), que analisou comparativamente diversos táxons de diferentes regiões tropicais, principalmente brasileiras, utilizando técnicas clássicas e modernas, pode ser mais representativo da evolução e padrão de diversificação cromossômica tropical (Veja também Ehrendorfer, 1982).

\section{Desenvolvimento da citotaxonomia no Brasil}

No Brasil, em particular, a abordagem citotaxonômica não teve maior receptividade entre os botânicos da primeira metade do século. Contudo, devido principalmente à presença de C. Krug no "Instituto Agronômico de Campinas" (IAC) e de F. Brieger na "Escola Superior de Agricultura Luiz de Queiroz" (ESALQ), melhoristas de várias culturas desenvolveram estudos citogenéticos para caracterizar o grupo de espécies (e eventuais híbridos) com que trabalhavam. Graner (1935), por exemplo, desenvolveu na ESALQ, em Piracicaba, a primeira análise de cromossomos mitóticos e meióticos de mandioca. Nesse trabalho inicial ele analisou 37 variedades brasileiras. Logo depois, o mesmo autor utilizou a técnica de indução de poliploidia por meio de colchicina para tentar aumentar a produtividade (Graner, 1941). Igualmente Bacchi (1940), do IAC, foi o primeiro a demonstrar a ocorrência de triplóides naturais em Citrus, e em seguida Krug (1943), fez uma extensa revisão da citogenética de Citrus e vários outros gêneros da subfamília Aurantioideae (Rutaceae). Estudos semelhantes foram desenvolvidos por Mendes (1938, 1939, 1946, 1947), também do IAC, em várias outras culturas, como café, amendoim, seringueira, algodão e fumo. Esses trabalhos ilustram bem a familiaridade dos melhoristas dessa época com a técnica citogenética. Com esse início nos centros de melho- 
ramento genético, não é de estranhar que a citogenética vegetal no Brasil tenha se desenvolvido predominantemente dentro dos Departamentos de Genética e só raramente nos Departamentos de Botânica. Mesmo os trabalhos iniciais com nativas sem interesse agronômico, como Iridaceae do gênero Alophia (Winge, 1959) ou Orquidaceae (Blumenschein, 1960), foram também desenvolvidos por geneticistas. Não é por acaso, que a maioria dos trabalhos recentes sobre número cromossômico em plantas, publicados no Brasil, se encontram na "Revista Brasileira de Genética" e não nas revistas de Botânica, embora o interesse maior desses estudos seja para botânicos e não para geneticistas. Noutros países a origem foi exatamente o inverso - a citogenética vegetal se desenvolveu dentro dos Departamentos de Botânica, como disciplina da Botânica.

\section{Situação atual da citotaxonomia no Brasil}

Atualmente a citogenética vegetal no Brasil continua voltada para as plantas cultivadas (veja, por exemplo, Moraes-Fernandes et al., 1985), inclusive devido aos problemas citogenéticos surgidos com o desenvolvimento das técnicas de cultura de tecidos (veja, por exemplo, Ferreira \& Handro, 1988). Contudo, há uma crescente tendência ao desenvolvimento de uma taxonomia com múltiplas abordagens, incluindo a análise citogenética. Exemplos disso podem ser observados tanto ao nível infragenérico, como em Axonopus (Hickenbick et al., 1975) e Eupatorium (Coleman \& Coleman, 1984, 1988), quanto ao nível supragenérico, como no complexo Phaseolus-Vigna-Macroptilium (Forni-Martins \& Da Cruz, 1985) ou em Chorisia-Ceiba (Gibbs et al., 1988). Além disso, surgem também pequenas listas de números cromossômicos, como as publicadas por Coleman $(1968,1970,1982)$, Coleman \& Smith (1969), Coleman \& De Menezes (1980), Maglio et al. (1984), Guerra (1986), Soares et al. (1988), Alves \& Custódio (1989) e Guerra \& Beltrão ( no prelo).

Embora a análise do número cromossômico continue sendo o principal instrumento da citotaxonomia, convém enfatizar que a análise citológica mais detalhada é necessária para permitir uma melhor visualização da variabilidade cariotípica real, e portanto deve ser tentada sempre que o material permitir. Entre os vários trabalhos nesse sentido podemos citar a análise comparada da morfologia cromossômica entre espécies de Briza (Schifino \& Winge, 1983) e Mikania (Ruas \& Ruas, 1987), do padrão cromomérico em cromossomos paquitênicos de espécies de Coffea (Pinto-Maglio \& Da Cruz, 1987), do padrão de bandas C em cultivares de milho (Aguiar-Perecin, 1985) ou o padrão de bandas fluorescentes em Eleutherine (Guerra, 1988).

É evidente que um maior entrosamento entre citogeneticistas e taxonomistas precisa ser ainda estimulado para propiciar o desenvolvimento dessa área no Brasil. Para isso, uma iniciativa importante seria a inclusão nos cursos 
de pós-graduação em Botânica criptogâmica e fanerogâmica, de uma disciplina de citogenética e citotaxonomia vegetal, com um programa prático objetivo, relacionado a projetos em desenvolvimentó ou ao estabelecimento de um projeto próprio voltado para um determinado gênero ou para determinada população. Por outro lado, seria igualmente importante que se estabelecessem meios específicos para garantir a publicação direta de pequenas listas de números cromossômicos em um periódico brasileiro de Botânica de ampla circulação. Isso provavelmente estimularia novas pesquisas nessa área e contribuiria para diminuir a carência de dados sobre a citogenética de nossa flora. É importante salientar, que espécies que já foram cariologicamente analisadas em uma determinada região não passam a ser menos interessantes para a análise por pesquisadores de outra região. Pelo contrário, a análise de várias populações de uma mesma espécie pode revelar desde a estabilidade citológica da espécie até a existência de espécies ou raças crípticas. Assim, a análise da morfologia cromossômica de Emilia sonchifolia nas populações de São José do Rio Preto (Coleman \& Campello, 1984) e de Recife (Guerra \& Nogueira, 1990) sugere pouca ou nenhuma diferenciação cariotípica dessa espécie durante sua dispersão pelo território brasileiro, enquanto a amostra de Genipa americana analisada em Pernambuco (Guerra, 1986), com 2n=22, parece diferir fortamente da de Campinas (Pierozzi \& Da Cruz, 1988), com $2 \mathrm{n}=20$.

Uma demonstração do crescente interesse pela citotaxonomia é o número crescente de laboratórios trabalhando nessa área e a diversidade de gêneros e famílias estudadas. A família com maior número de estudos é a das leguminosas (Porto Alegre - Trifolium, Desmodium, Leucaena; Santa Maria - Centrosema; Piracicaba - Stylosanthes; Campinas - Phaseolus, Vigna, Macroptilium; Salvador - Crotalaria; Recife - Caesalpinia; São José do Rio Preto, Piracicaba, Recife e Fortaleza - número cromossômico em diversos gêneros). As gramíneas têm também recebido especial atenção em Porto Alegre (Paspalum, Axonopus, Briza, Setaria, Hordeum, além dos trigos, centeios e triticales em um centro de pesquisas próprio em Passo Fundo CNPT), Piracicaba (Saccharum) e Goiânia (Brachiaria e Hyparrhenia), além de um trabalho extenso com milho em Piracicaba e, mais recentemente, em Maringá. Igualmente as Compositae vêm sendo estudadas em vários centros, como em São José do Rio Preto (Emilia, Verbesina, Eupatorium e outros gêneros em listas de números cromossômicos), Londrina (Mikania, Eupatorium, Baccharisł) e Recife (Emilia e outros gêneros em listas de números cromossômicos). Além disso, há também algumas famílias menores (Bombacaceae em Campinas; Velloziaceae em São Paulo/Recife; Rutaceae em Recife) e um grande número de gêneros e espécies isoladas (Nothoscordum, Talauma, Clusia, Costus, Zephyranthes, Hippeastrum, Thunbergia, Aptenia, Ilex, Dichorisandra, Passiflora, Cattle$y a$, etc.) que vêm sendo estudados em diversos laboratórios. No caso das famílias investigadas independentemente por diversos grupos, como as leguminosas, uma ação coordenada que permitisse estabelecer um projeto geral e os 
meios de colaboração entre pesquisadores, certamente ampliaria em muito o nível dos resultados que vêm sendo obtidos. De qualquer forma, é evidente a necessidade e a tendência da citogenética de se introduzir e se expandir dentro da Botânica, permitindo consolidar o desenvolvimento da citotaxonomia no Brasil.

\section{Referências bibliográficas}

AGUIAR-PERECIN, M.L.R. 1985. Bandeamento C e tipos de heterocromatina em milho. In: M.L.R., AGUIAR-PERECIN, P.S. MARTINS e G. BANDEL eds. Tópicos de Citogenética e Evolução de Plantas. Sociedade Brasileira de Genética. Ribeirão Preto, SP. p.51-67.

ALVES, M.A.E. \& A.V.C. CUSTÓDIO 1989. Citogenética de Leguminosas coletadas no Estado do Ceará. Rev. Brasil. Genet. 12;81-92.

BACCHI, D. 1940. Observações citológicas em Citrus. I. Número de cromossomos de algumas espécies e variedades.. Jornal de Agronomia, Piracicaba 3: 249-258.

BARLOW, B.A. 1959. Chromosome numbers in the Casuarinaceae. Austral. J. Bot. 7: 230-237.

BLUMENSCHEIN, A. 1960. Número de cromossomos de algumas espécies de orquídeas. Publ. Cient. Univ. São Paulo 1: 47-48.

Böcher, T. W. 1961. The development of cytotaxonomy since darwin's time. In: P.J. WANSTALL, ed. A Darwin centenary. B.S.B.I. - Conference Reports, Number six, London. p.26-43.

BRANDHAM, P.E. 1988. Kew Chromosome Conference III. Her Majesty's Stationery Office, London.

COLEMAN, J. 1968. Chromosome numbers in some Brazilian Compositae. Rhodora 70 : 228-240.

COLEMAN, J. 1970. Additional chromosome numbers in Brazilian Compositae. Rhodora 72: 94-99.

COLEMAN, J. 1982. Chromosome numbers of angiosperms collected in the State of São Paulo. Rev. Brasil. Genet. 5; 533-549.

COLEMAN, J. \& M.L. CAMPELLO 1984. Cytogenetics of a mixed Brazilian population of Emilia sonchifolia (L.) DC. and. E. coccinea (Sims) G. Don (Compositae). Rev. Brasil, Genet. 7: 83-94.

COLEMAN, J.R. \& M.A. COLEMAN 1984. Apomixis in two triploid Brazilian species of Eupatorium: E. bupleurifolium and E. Callilepis. Rev. Brasil. Genet. 7: 549-567.

COLEMAN, J.R. \& M.A. COLEMAN. 1988. Embryology and cytogenetics of apomictic triploid Eupatorium squalidum DC. (Compositae). Rev. Brasil. Genet. 11: 129-148.

COLEMAN, JR. \& E.M. MENEZES. 1980. Chromosome numbers in Legu- 
minosae from the State of São Paulo, Brazil. Rhodora. 82: 475-481.

COLEMAN, J. \& L. SMITH. 1969. Chromosome numbers of some Brazilian angiosperms. Rhodora. 71:548-551.

DARLINGTON, C.D. 1956. Chromosome Botany. George Allen \& Unwin Ltd., London.

DELAY, C. 1949. Recherches sur la structure des noyaux quiescents chez les phanérogames. Masson \& Cie, Editeurs Paris.

DIERS, L. 1961. Der Anteil an Polyploiden in den Vegetationsgürteln der Westkordillere Perus. Zeitschr. Bot. 49: 437-488.

EHRENDORFER, F. 1964. Cytologie, Taxonomie und Evolution bei Samenpflanzen. Vistas in Botany 4: 99-186.

EHRENDORFER, F. 1970. Chromosomen, Verwandtschaft und Evolution tropischer Holzpflanzen. I. Allgemeine Hinweise. Österr. Bot. Z. 118: 30-37.

EHRENDORFER, F. 1982. Speciation patterns in woody angiosperms of tropical origin. In: C. BARIGOZZI. ed. Mechanisms of Speciation. Alan R. Liss, Inc., New York. p.479-509.

FAVARGER, C. 1978. Philosophie des comptages de chromosomes. Taxon, 27: 441-448.

FEDOROV, A.M.A. 1969. Chromosome Number of Flowering Plants. Komarov Botanical Institute, Leningrado.

FERREIRA, C.M. \& W. HANDRO. 1988. Production, maintenance and plant regeneration from cell suspension cultures of Stevia rebaudiana (Bert.) Bertoni. Pl. Cell Rep. 7: 123-126.

FORNI-MARTINS, E.R. \& N. D. CRUZ. 1985. Estudos citotaxonômicos no complexo Phaseolus-Vigna-Macroptilium (Leguminosae-Papilionoideae). In: M.L.R. AGUIAR-PERECIN, P.S. MARTINS E G. BANDEL, eds. Tópicos de Citogenética e Evolução de Plantas. Sociedade Brasileira de Genética. Ribeirão Preto, SP. p. 155-172.

GIBBS, P.E., J. SEMIR \& N.D. CRUZ. 1988. A proposal to the unite the genera Chorisia Kunth and Ceiba Miller (Bombacaceae). Notes R.B.G. Edinb. 45: 125-136.

GOLDBLATT, P. 1981. Index to Plant Chromosome Number 1975-1978. Missouri Botanical Garden, St. Louis.

GOLDBLATT, P. 1984. Index to Plant Chromosome Number 1979-1981. -Missouri Botanical Garden, St. Louis.

GOLDBLATT, P. 1985. Index to Plant Chromosome Number 1982-1983. Missouri Botanical Garden, St. Louis.

GOLDBLATT, P. 1988. Index to Plant Chromosome Number 1984-1985. Missouri Botanical Garden, St. Louis.

GRANER, E.A. 1935. Contribuição para o estudo citológico da mandioca. Escola Superior de Agricultura "Luiz de Queiroz", Piracicaba, São Paulo. 
GRANER, E.A. 1941. Polyploid cassava induced by colchicine treatment. $J$. Heredity 32: 281-288.

GRANT, W.F. 1955. A cytogenetic study in the Acanthaceae. Brittonia 8: 121-149.

GRANT, W.F. 1984. Plant Biosystematics. Academic Press. Ontario.

GREILHUBER, J. 1984. Chromosomal evidente in taxonomy. In: V.H. HEYWOOD and D.M. MOORE, eds. Current Concepts in Plant Taxonomy. Academic Press, London. p. 157-180.

GUERRA, M.S. 1985. Estrutura e diversificação dos núcleos interfásicos em plantas. In: M.L.R. AGUIAR-PERECIN, P.S. MARTINS e G. BANDEL, eds. Tópicos de Citogenética e Evolução de Plantas. Sociedade Brasileira de Genética. Ribeirão Preto, SP. p. 137-153.

GUERRA, M.S. 1986 Citogenética de angiospermas coletadas em Pernambuco. I. Rev. Brasil. Genet. 9: 21-40.

GUERRA, M.S. 1987. Cytogenetics of Rutaceae IV. Structure and systematic significance of interphase nuclei. Cytologia 52: 213-222.

GUERRA, M.S. 1988. Mitotic and meiotic analysis of a pericentric inversion associated with a tandem duplication in Eleutherine bulbosa. Chromosoma 97: 80-87.

GUERRA, M.S. \& G.T.A. BELTRÃO. Citogenética de angiospermas coletadas em Pernambuco. III. Ci. e Cult. (no prelo)

GUERRA, MS. \& M.T.M. NOGUEIRA 1990. The cytotaxonomy of Emilia spp. (Asteraceae: Senecioneae) occurring in Brazil. Pl. Syst. Evol. 170: 229-236.

HICKENBICK, M.C.M., J.F.M. VALLS, F.M. SALZANO \& M.I.B. MORAES-FERNANDES. 1975. Cytogenetic and evolukionary relationship in the genus Axonopus (Gramineae). Cytologia 40: 185-204.

JACKSON, R.C. 1976. Evolution and systematic significance of polyploid. Ann. Rev. Syst. 7: 209-234.

JOHNSON, L.A.S. \& B.G. BRIGGS. 1963. Evolution in the Proteaceae. Aust. J. Bot. 11: 21-61.

JONES, K. \& A. KENTON. 1984. Mechanisms of chromosome change in the evolution of the tribe Tradescantieae. (Commelinaceae). In: A. SHARMA, and A.K. SHARMA, ed. Chromosomes in Evolution of Eukaryotic Groups II. C.R.C. Press, Boca Raton. p. 143-168.

KRUG, C.A. 1943. Chromosome number in the subfamily Aurantioideae: with special reference to the genus Citrus. Bot. Gaz. 104: 602-611.

LEVIN, D.A. \& S.W. FUNDERBURG. 1979. Genome size in angiosperms: temperate versus tropical species: Am. Nat. 144: 784-795.

LEWIS, W.H. 1980. Polyploidy, Biological Relevance. Plenum Press, New York.

MAGLIO, C.A.F.P., E.R. FORNI-MARTINS \& N.D. CRUZ. 1984. In: A. 
LÖVE, ed. IOPB Chromosome Number Reports LXXXIV. Taxon 33: 536-539.

MANGENOT, S. \& G. MANGENOT. 1962. Enquête sur les nombres chromosomiques dans une collerction d'espèces tropicales. Rev. Cyt. et Biol. Vég. 25: 411-447.

MANNA, G.K. \& U. SINHA. 1981 Perspectives in Cytology and Genetics. Vol. 3. Hindasia Publishers, New Delhi.

MEHRA, P.N. 1976. Cytology of Himalayan Hardwoods. - Sree Saraswaty Press Ltd. Calcutta.

MENDES, A.J.T. 1938. Morfologia dos cromossômos de Coffea excelsa Chev. Bol. Técn. Inst. Agron. Est. Campinas, 56: 3-10.

MENDES, A.J.T. 1939. Duplicação do número de cromossômos em café, algodão e fumo pela ação de colchicina. Bol. Técn. Inst. Agron. Est. Campinas 57: 3-21.

MENDES, A.J.T. 1946. Investigações preliminares sobre a duplicação do número de cromossômios da seringueira pela ação da colchicina. Bol. Técn. Inst. Agron. Norte 7: 1-60.

MENDES, A.J.T. 1947. Estudos citológicos no gênero Arachis. Bragantia 7: 257-267.

MERXMÜLLER, H. 1970. Provocation of biosystematics. Taxon 19: $140-145$.

MOORE, R.J. 1973. Index to plant chromosome number 1967-1971. Regnum Vegetabile 90: 1-539.

MOORE, R.J. 1974. Index to plant chromosome number 1972. Regnum Vegetabile 91: 1-108.

MOORE, R.J. 1977. Index to plant chromosome numbers for 1973-1974. Regnum Vegetabile 96: 1-257.

MORAES-FERNANDES, M.I.B., M.H.B. ZAN TTINI, M.S. GUERRA, L.J.A. DEL DUCA, M.J.C. SERENO \& C.C. ZANELLA. 1985. Instabilidade cromossômica e adaptação em trigo. In: M.L.R. AGUIAR-PERECIN, P.S. MARTINS \& G. BANDEL eds. Tópicos de Citogenética e Evolução de Plantas. Sociedade Brasileira de Genética, Ribeirão Preto, SP. p. 69-123.

MORAWETZ, W. 1986. Remarks on karyological differentiation patterns in tropical woody plants. $\mathrm{Pl}$. Syst. Evol. $152: 49.100$.

MORTON, J.K. 1966. The role of polyploidy in evolution of a tropical flora. Chromosomes Today 1: 73-76.

PIEROZZI, N.I. \& N.D. CRUZ. 1988. Número e identificação dos cromossomos de Genipa americana L. (Rubiaceae) através de técnica de banda-C. Ci. e Cult. 40 (Supl.): 802.

PINTO-MAGLIO, C.A.F. \& N.D. CRUZ. 1987. Pachytene chromosome morphology in Coffea L.I. Nucleolar chromosomes. Caryologia 40; 7-23. 
RAVEN, P. 1975. The bases of angiosperm phylogeny: Cytology. Ann. Missouri Bot. Gard. 62: 724-764.

REESE, G. 1957. Uber die Polyploidiespektren in der nordsaharischen Wüstenpflanzen. Flora 144: 598-634.

RUAS, P.M. \& C.F. RUAS. 1987. Karyontypes and chromosome morphology in the genus Mikania (Compositae) I. Cytologia 52: 551-558.

SCHIFINO, MT. \& H. WINGE. 1983. Karyotypes and nuclear DNA content of species of the Briza complex and some other genera of Poeae (Gramineae). Rev. Brasil, Genet. 6:245-249.

SOARES, M.M.M., M.S. GUERRA \& F. GALLINDO. 1988. Citogenética de angiospermas coletadas em Pernambuco - II Ci. e Cult. 40: 780-786.

SMITH-WHITE, S. 1954. Chromosome numbers in the Boronieae (Rutaceae) and their bearing on the evolutionary development of the tribe in the Australian flora. Austral. J. Bot. 2: 287-303.

SMITH-WHITE, S. 1959. Cytological evolution in the Australian flora. Cold Spring Harb. Symp. Quant. Biol. 24: 273-289.

STACE, C.A. 1984: Plant Taxonomy and Biosystematics. Edward Amold. Avon.

STEBBINS, G.L. 1966. Chromosome variation and evolution. Science 152: 1463-1469.

STEBBINS, G.L. 1971. Chromosomal Evolution in Higher Plants. Edward Arnold, London.

WINGE, H. 1959. Studies on cytotaxonomy and polymorphism of the genus “Alophia' (Iridaceae), Rev. Brasil. Biol. 19: 195-201. 